\title{
Test Flow Selection for Stacked Integrated Circuits
}

\author{
Breeta SenGupta $^{1} \cdot$ Dimitar Nikolov $^{1} \cdot$ Assmitra Dash $^{2} \cdot$ Erik Larsson $^{1}$
}

Received: 22 June 2018 / Accepted: 16 May 2019 / Published online: 14 August 2019

(C) The Author(s) 2019

\begin{abstract}
Integrated circuits (ICs) with a single chip (die) are typically tested with a test flow consisting of two test instances: (1) wafer sort for the bare chip and (2) package test for the packaged IC. For ICs with stacked chips - 3D Stacked ICs - there are many possible test instances, even more test flows, and no commonly used test flow. In this paper, we propose a test flow selection algorithm (TFSA) to obtain a test flow for a given 3D Stacked IC. The TFSA results in a test flow for a given 3D Stacked IC, such that the expected total test time to produce each good package is minimized. We implemented the TFSA, three straightforward test flow schemes and an exhaustive search, and experimentally compared the test flow schemes on three different test architecture design approaches. The results demonstrate the importance to have methods both to select the test flow and design the test architecture.
\end{abstract}

Keywords 3D IC · Stacked integrated circuits · Test flow · Test time · Yield · Test plan · IEEE $1500 \cdot$ Test architecture . Expected time $\cdot$ Effective yield $\cdot$ Quantity

\section{Introduction}

The constant development in semiconductor technologies enables increasingly advanced integrated circuits (ICs). Today, it is possible to manufacture wafers where each individual chip (die) contains billions of transistors. After manufacturing, the chips are first cut from the wafer and then wire bonded to connect the chip to the package. Finally, the chips are packaged. The most recent advancement in semiconductor technologies is to stack several chips on top

Responsible Editor: E. J. Marinissen

Erik Larsson

erik.larsson@eit.lth.se

Breeta SenGupta

breetasg@gmail.com

Dimitar Nikolov

dimitar.nikolov@altran.com

Assmitra Dash

asmitra.dash@gmail.com

Lund University, Lund, Sweden

2 Linköping University, Linköping, Sweden of each other and package them in one IC - 3D Stacked IC. The chips in such a 3D Stacked IC are connected by through silicon vias (TSVs) [8].

IC manufacturing is extremely complex, which increases the risk of defects. To detect manufacturing defects, each and every IC is carefully tested. ICs with a single chip are commonly tested with a test flow consisting of two test instances; wafer sort and package test. The bare chip is tested at wafer sort to avoid packaging of defective chips. If no defects are found at wafer sort, the chip is wire-bonded, packaged, and re-tested during package test as defects may be introduced during wire-bonding and packaging. For 3D Stacked ICs there are many more test instances. It is possible to test each individual chip during wafer sort instances, at intermediate test instances where the partially complete stacks can be tested, and at package test instance where the complete 3D Stacked IC is tested. For a 3D Stacked IC with $N$ chips there are $2 N$ instances when a test may be performed, $N$ during wafer sort, $N-1$ for intermediate stacks and 1 for package test. Hence, there are $2^{2 N}$ possible variations of test flows [8].

The test cost of 3D Stacked ICs depend on a large number of factors, such as the hardware manufacturing cost that includes wafer fabrication, stacking and packaging, DfT (design for test), fault coverage, test resource and test equipment, the test time and yield. In this paper we reduce the test cost per good 3D Stacked IC produced, by selecting 
a test flow optimizing two of the major contributing factors: test time and yield. Eventually, we address minimization of the expected test time for each good 3D Stacked IC produced, and implement that along with test architecture optimization as described in previous articles $[12,13]$.

The expected test time may vary both with the choice of test flow as well as the applied test schedule. The time spent on testing defective ICs also contributes to the test cost. Hence, the manufacturing yield needs to be taken into account. For 3D Stacked ICs, where it is possible to stack a number of different chips, it is of interest to know how many chips of different types are needed in the manufacturing process to obtain a fixed number of good packages of 3D Stacked IC.

The test cost can be reduced by improving the yield and/or by reducing the time spent on testing. Improving yield implies reducing defects in the manufacturing process, for example, by upgrading production technologies. Yield improvement is not in the scope of this paper. In this paper, we focus on minimizing the time spent on testing.

In this paper, we assume a 3D Stacked IC, where the test time and yield at each test instance is known. With the goal of minimizing the expected test time per good 3D Stacked IC package, we propose a method to compute the effective yield, the number of chips that need to be tested at each test instance, and the expected test time, depending on the selected test flow. To find the most suitable test flow, we propose the Test Flow Selection Algorithm (TFSA). We performed experiments on several 3D Stacked ICs to compare the test flow obtained using TFSA against test flows obtained by exhaustive search and three straightforward test flows - wafer sort of each individual chip followed by package test (WSPT), test at all possible instances (TA) and test performed only at package test (PT). The results demonstrate that (1) TFSA produces results that are better than the three straightforward test flow schemes, (2) TFSA produces optimal test flow in most cases, and (3) the straightforward test flow where wafer sort of each chip is followed by package test of the complete 3D Stacked IC gives the best result among the three straightforward test flow schemes. We also integrated test flow selection with test architecture design, adopting the test planning scheme proposed in [12]. While [12] optimizes the test architecture for a single test flow that consists of wafer sorts of the individual chips followed by package test of the complete 3D Stacked IC, in this paper we generalize the approach for any given test flow. The experimental results validate that it is important to have methods to find the test flow as well as methods to design the test architecture. The test flow model and TFSA complies with all die orientations - face-to-face, face-to-back and back-to-back; as well as all wafer bonding technologies - wafer-to-wafer, die-to-die and die-to wafer.
The limitations of this work are as follows. First, we assume that all test flows include package test. This is motivated by the fact that if the final test instance (package test) is not performed, all defects in the last test instance are not checked. Hence it is self-evident to assume package test to be mandatory. Second, in our experiments we calculate the test time of a intermediate stage as the sum of the test times of each individual die in the partial stack and that of the interconnects. Thus, each die would undergo the same test during wafer sort and all successive intermediate stages. The yields at all intermediate stages are also assumed to be equal during the experiments. Third, in this paper we address reduction of a part of the test cost by minimizing the test time associated with test flow selection. Optimization of all factors contributing to the test cost, like manufacturing cost or fault coverage, would lead to even higher complexity to the problem of selecting the most suitable test flow, and have therefore not considered in this paper. The expressions are however scalable to accommodate the trade-off with additional contributing factors, which is addressed in our future work.

The rest of the paper is organized as follows. In Section 2 we discuss related research. The test architecture is elaborated in Section 3. In Section 4, we illustrate with an example at three different yield sets the need of finding a suitable test flow. In Section 5, we introduce notations and formulae, while in Section 6 we present the TFSA. In Section 7 we report the results from the experiments. The paper is concluded in Section 8.

\section{Related Work}

Several works have addressed test planning for core-based ICs having a single chip with the aim of optimizing the test cost $[2,6,7]$. Design and optimization of test architecture for non-stacked ICs with IEEE 1500 is described in [4, 5, $11,15]$. In [5], Iyengar et al. address optimization of test access mechanisms (TAMs) for System-on-Chips (SoCs) to reduce core-test time by balancing core scan chains. Mullane et al. in [11] propose a hybrid scan for non-stacked ICs provided with IEEE 1500 core wrappers, by combining the serial and the parallel ports of the wrapper, resulting in an efficient test access that reduces the test time. However, for 3D Stacked ICs, test architecture optimized for each chip in the stack during wafer sort may not lead to an optimized test architecture when all the chips are tested jointly during package test.

We have proposed methods to reduce the test time for core-based 3D Stacked ICs, by optimizing the test architecture and the test plan [12, 13]. We used a straightforward test flow, where each individual chip is tested at wafer sort and the complete stack at package test. 
The test planning approaches were not adapted for arbitrary test flows.

Taouil et al. propose test cost models to predict the impact of test flows on the product quality and overall stack cost at an early design stage, which is important for a trade-off between quality and cost [14]. They present a model that predicts the product quality, defined in terms of Defective Parts Per Million (DPPM) for different test flows. A framework is provided for covering different test flows and cost models to identify the most cost effective test flow [3]. Simulation results show that test flows that include wafer sort generally reduce the overall cost and that the most cost-effective test flow strongly depends on the stack yield. They concluded, after experiments on different test flows, that adapting the tests according to the stack yield is a good approach. The paper analyzes several test flows for given 3D Stacked ICs, but do not provide a method for the selection of the most suitable test flow. Agrawal et al. in [1] have proposed a low complexity test flow selection scheme for 3D Stacked ICs to achieve a low test cost. It is shown that the test flow selection method takes considerably lower computation time as compared to exhaustive methods that completely explore the exponentially growing search space for 3D Stacked ICs. However, an estimate of the margin of the increase in test cost by using the proposed method against exhaustive search is lacking. Both [1, 14] are built on the compromise between yield and test cost. However, the trade-off among two major contributing factors to the test cost, namely, test time and manufacturing cost of each component has been overlooked. The work is not verified against any test architecture design and test planning scheme. To the best of our knowledge, no work has previously proposed a test flow selection algorithm and verified it against any test architecture and test planning scheme.
A new test standard, IEEE P1838 is being developed to enable efficient modular testing of SICs [10]. The standard involves a die level wrapper on each chip in the stack. In addition, an IEEE 1149.1 based TAP controller is provided in the bottom die that controls the WIRs of the die wrappers. [12, 13] addresses test scheduling for SICs to minimize the test cost. In [12] a scalable test architecture is assumed where each chip is provided with a IEEE 1500 based wrapper, in accordance with the developing IEEE P1838 standard. Similarly, [13] assumes a IEEE 1149.1 based test architecture for the SICs for test planning.

\section{IEEE 1500 Based Test Architecture}

In this section we discuss the test architecture for a 3D Stacked IC, where each chip of the stack is supported by a IEEE 1500 based infrastructure as proposed by [8].

A number of chips are stacked to construct a 3D Stacked IC. Figure 1 shows a 3D Stacked IC with two chips, where Chip2 is stacked on top of Chip1, where each chip comprises of several cores. Chip1 contains core1, core2 and core3, while Chip2 hosts core 4 and core5. To enable testing, each core consists of a number of scan-chains that are concatenated over a set of TAM lines to form wrapperchains which are connected to the wrapper. The Wrapper Parallel Port (WPP) and the Wrapper Serial Port (WSP) constitute the test terminals for the 3D Stacked IC. The WPP includes the Wrapper Parallel Input (WPI) and the Wrapper Parallel Output (WPO), each with a width $W$, that is decided by the design er. The WSP, comprises of Wrapper Serial Input (WSI), Wrapper Serial Output (WSO), and Wrapper Serial Control (WSC) terminals, and supports serial test mode. The instruction to be executed in a chip is stored in the corresponding Wrapper Instruction Register (WIR).

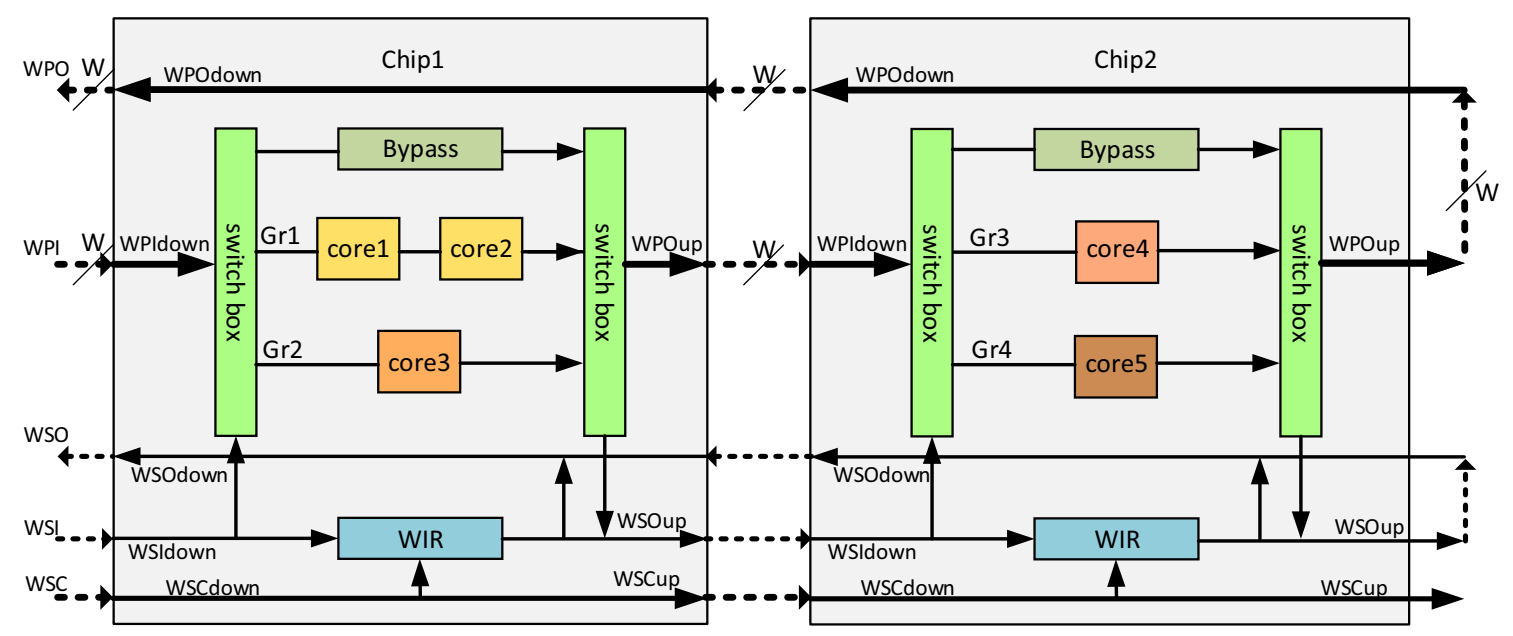

Fig. 1 Test architecture of a 3D Stacked IC, with two chips in the stack: Chip2 stacked on Chip1, where each chip is supported by the IEEE 1500 based test architecture 
Within each chip, the TAM width, WPI, is split at the switch box, to several groups of TAMs. Each group of TAM is used to access one or more cores of the chip, connected in series. The TAM width $W$ in Fig. 1, for Chip1 is split among $G r_{1}$ connecting core 1 and core2 in series, and $G r_{2}$ to core3, while for Chip2 $G r_{3}$ and $G r_{4}$ accesses core4 and core5, respectively. Wrapper-chains are constructed by allocating one or more scan-chains to a single TAM line. In Fig. 2, core1 of Chip1 is illustrated, that has three scanchains: $s c 1, s c 2$ and $s c 3$. TAM group $G r 1$ is used to access core1, such that $s c 1$ forms a wrapper-chain on $T A M 1$, while $s c 2$ and $s c 3$ in series is a wrapper-chain on $T A M 2$. As illustrated in Fig. 1. the WPOup, WSOup and WSCup of the lower chip, Chip1, is connected to the WPIdown, WSIdown and WSCdown of the chip on top, Chip2, respectively. The WPOup, WSOup and WSCup of the topmost chip, Chip2, are directed out via the WPOdown, WSOdown and WSCdown, respectively, of the lowermost chip in the stack, Chip1. The WPOdown, WPIdown, WSOdown, WSOdown and WSCdown of the lowermost chip, Chip1, serve as the package test interface for the 3D Stacked IC. As in [9], in this paper we assume equal width of WPIs and WPOs for each chip.

The TSV interconnect between chips may be tested using the boundary scan registers, which connects all input/output via TSVs. Boundary scan registers are implemented on both chips and are used in TSV interconnect test. Test stimuli are applied on out-going TSVs and test responses are captured on in-coming TSVs. Since the boundary scan register is a separate register, testing of TSVs cannot be performed concurrently with core tests.

The TSV interconnect tests contribute with a constant term to the overall test time and could not be scheduled with any core tests. Therefore, the time required to perform TSV interconnect tests are overseen while addressing the total test time in the remainder of the paper.

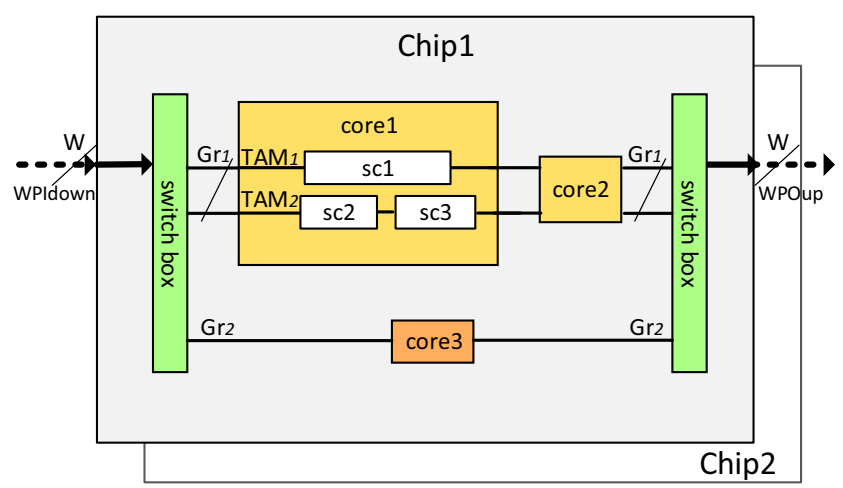

Fig. 2 Scan-chains configured into wrapper-chains in core1 of Chip1

\section{Impact of Test Flow on the Expected Test Time}

In this section we show with an example the effect of test flow on the expected total test time. The example considers a 3D Stacked IC with two chips. Four test instances exist for the 3D Stacked IC, viz, wafer sort of each individual chip (WS1 and WS2, respectively), intermediate test (IT) of the two chips, and package test (PT) of the final 3D Stacked IC.

Table 1 details the given testing times for each instance. To demonstrate the impact of test flows, we consider three sets of yield values for each test instance, as shown in Table 1. For example, for yield Case 1, the yield at the wafer sort of Chip 1 is 0.90 .

We use three straightforward test flow schemes:

- Test all (TA): tests are applied at every possible test instance;

- Wafer sort and package test (WSPT): each individual chip is tested at wafer sort and the complete 3D Stacked IC is tested at package test;

- Package test (PT): testing is only applied to the complete 3D Stacked IC at the final test instance, package test;

We compare the expected total test time required to obtain each good 3D Stacked IC, by assuming TA, WSPT and PT as the test flows. Figure 3 illustrates the expected total test time required by the three test flows with the three sets of yield values. Computation details of the expected test time required by the test flows are elaborated in the following Section.

The results show that for Case 1 PT has the lowest expected test time, while in Case 2, the test flow with the lowest expected test time is WSPT, and that in Case 3 TA results in the lowest expected test time. Thus, it can be concluded from the results that a straightforward test flow may not provide the lowest expected test time for any given 3D Stacked IC. In this paper we present a method to obtain a test flow for any given 3D Stacked IC, such that the expected total test time is minimized.

Table 1 SIC with three different sets of yield

\begin{tabular}{lllll}
\hline Test instance & WS1 & WS2 & IT & PT \\
\multicolumn{1}{c}{$I_{i j}$} & $I_{11}$ & $I_{21}$ & $I_{22}$ & $I_{32}$ \\
\hline Test time $T_{i j}$ & 10 & 10 & 30 & 70 \\
Yield $y_{i j}$ (Case 1) & 0.90 & 0.91 & 0.92 & 0.93 \\
Yield $y_{i j}$ (Case 2) & 0.70 & 0.71 & 0.72 & 0.73 \\
Yield $y_{i j}$ (Case 3) & 0.50 & 0.51 & 0.52 & 0.53 \\
\hline
\end{tabular}




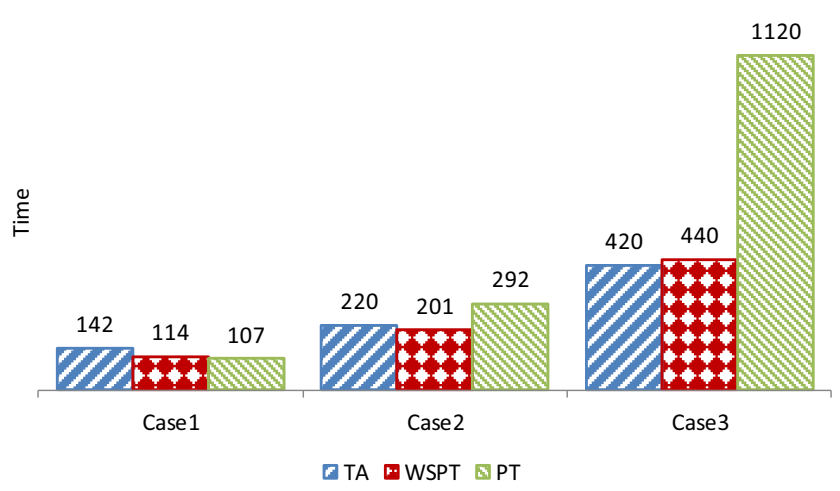

Fig. 3 Comparison of expected total test times for three test flows TA, WSPT and PT respectively - on one design with three sets of yield values: Case 1, Case 2, and Case 3. PT, WSPT and TA require the lowest expected test times for Case 1, Case 2 and Case 3 respectively

\section{Expected Total Test Time Estimation}

In this section we derive an expression to calculate the expected total test time required to produce each fault-free 3D Stacked IC for any assumed test flow. For reference, we assume the design provided in Table 1 with yield Case 1.

We elaborate the notations given in Table 2 using Figs. 4 and 5. First, the given notations corresponding to given data are discussed, followed by the notations that refer to values that need to be calculated for a selected test flow. Finally, we discuss the notations that represent values that hold true for all 3D Stacked ICs considered in this paper. We assume a given 3D Stacked IC with $N$ chips in the stack, where each chip is denoted by $i, 1 \leq i \leq N$. A test instance is denoted by $I_{i j}$, illustrated in Fig. 5 . Each test instance, $I_{i j}$, requires test time $T_{i j}$ and has a yield $y_{i j}$.

The wafer sort instances are illustrated by the boxes in the upper row, where $j=1$, which means $I_{11}$ to $I_{N 1}$ are the instances for wafer sort. The wafer sort instance of a chip $i$ is indicated with $I_{i 1}$ to the left in Fig. 4. We have $j=2$ for intermediate tests of partial stacks with $i$ chips, and for package test instances, $I_{i 2}$. In Fig. 5, the bottom row indicates the intermediate test instances and the package test. For an intermediate test instance $I_{i 2}$, the intermediate stack consists of all chips from 1 to $i$. For example, if $i=3$, the intermediate test instance $I_{32}$ consists of the partial stack of chip 1, 2 and 3. The intermediate test instances and package test are illustrated to the right in Fig. 4.

A partial stack, with $i$ chips, is tested during the intermediate instance, $I_{i 2}$, which comprises of components from two previous instances - a partial stack with $i-1$ chips, and chip $i$. This is illustrated by arrows connecting instance $I_{i-12} \rightarrow I_{i 2}$, and $I_{i 1} \rightarrow I_{i 2}$, in Fig. 5 .

We now discuss the values that need to be computed to obtain the desired test flow. A test flow is represented by the vector $X=\left(x_{11} \ldots x_{N 1}\right),\left(x_{22} \ldots x_{N 2}\right),\left(x_{N+12}\right)$, with $2 N$ elements, that include $N$ wafer sorts, $N-1$ intermediate tests and 1 package test. Each element is denoted by the binary decision variables $x_{i j}$, for each box in Fig. 5, we set $x_{i j}=1$ when a test is performed at instance $I_{i j}$, and $x_{i j}=0$ when no tests are performed at instance $I_{i j}$. Let us consider the example of a 3D Stacked IC with 2 chips in the stack, as illustrated in Section 4. The vectors for TA, WSPT and PT would be represented as: $X=(1,1),(1),(1)$, $X=(1,1),(0),(1)$ and $X=(0,0),(0),(1)$ respectively. For convenience, we also define $\bar{x}_{i j}=1-x_{i j}$.

$\tau$ is the total expected time taken by the test flow given by $X$. The total expected time depends on what tests are applied in a test flow. At each instance, the effective yield is computed depending on the tests that have been previously performed. To enable computation of test time, for a test instance $I_{i j}$, we let $Q_{i j}$ denote the number of good units that need to be produced at instance $I_{i j}, T_{e f f}(i j)$ denote the expected time taken to produce each good unit at instance $I_{i j}$, and $Y_{i j}$ the effective yield. The objective of this paper is to minimize the expected total test time $\tau$.

Finally, it is given for all 3D Stacked ICs that, instances $I_{12}$ and $I_{N+11}$ do not exist. Instance $I_{12}$ corresponds to the box at the bottom left of Figure 5, for the intermediate test of only chip 1, which does not exist as at least two stacked chips are tested at any intermediate test. Again, for a 3D Stacked IC comprising of $N$ chips in the stack, instance $I_{N+11}$ corresponding to the box at the top right corner of Fig. 5, refers to wafer sort of chip $N+1$, also does not exist. Therefore, it may be assumed that these instances require no test time, i.e., $T_{12}=T_{N+11}=0$, and also have a perfect yield, $y_{12}=y_{N+11}=1$, for the generic expressions.

In the following Sections 5.1, 5.2, and 5.3 respectively, we elaborate each component of the expression, viz, yield $Y_{i j}$, quantity $Q_{i j}$ and time $T_{e f f}(i j)$.

\subsection{Yield}

The effective yield at each test instance is presented here, which is given as a function of the given yield values of the preceding test instances, depending on whether a test was performed during that instance. We will discuss effective yield first for wafer sort and then for intermediate and package tests.

Let us consider an arbitrary wafer sort test instance $I_{i 1}$, which has the given yield of $y_{i 1}$. As there are no prior tests of the chip at wafer sort, the effective yield depends only on the yield at test instance $I_{i 1}$.

For wafer sort instances the effective yield $Y_{i 1}$ at test instance $I_{i 1}$ is given as:

$Y_{i 1}=y_{i 1}$ 
Table 2 List of notations

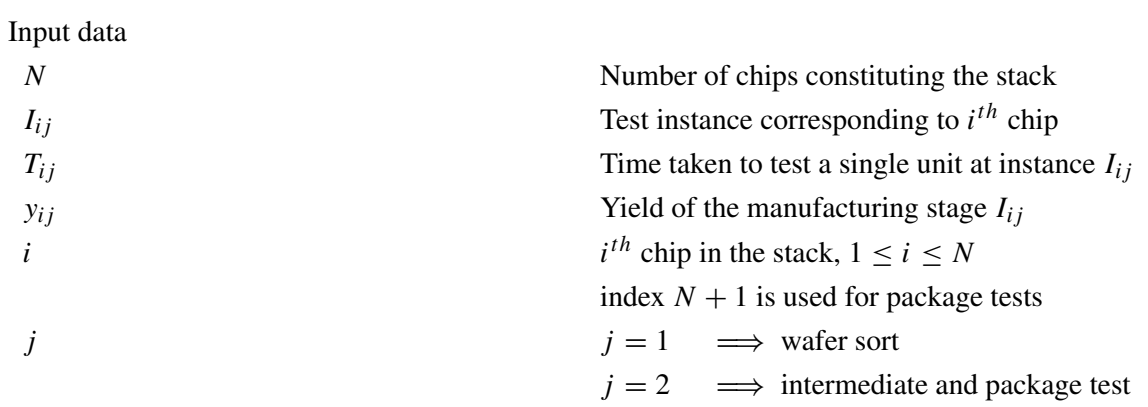

Known data

$I_{12}$

$I_{N+11}$

$Q_{N+12}$

$Q_{i 2}$

Calculated

$x_{i j}$

$\bar{x}_{i j}$

X

$Q_{i j}$

$T_{e f f}(i j)$

$Y_{i j}$

$\tau$
Does not exist

$T_{12}=0$

$y_{12}=1$

Does not exist

$T_{N+11}=0$

$y_{N+11}=1$

$=1$

$=Q_{i-12}$

Binary decision variable

1 ; if test is performed at instance $I_{i j}$

0 ; otherwise

$1-x_{i j}$

Test flow vector composed of $x_{i j}$ $X=\left(x_{i 1} \mid 1 \leq i \leq N\right),\left(x_{i 2} \mid 2 \leq i \leq N\right),\left(x_{N+12}=1\right)$

Number of good units that need to be produced at instance $I_{i j}$

Expected time taken to produce each good unit at instance $I_{i j}$

Effective yield at instance $I_{i j}$

Expected total test time taken by the test flow given by $X$

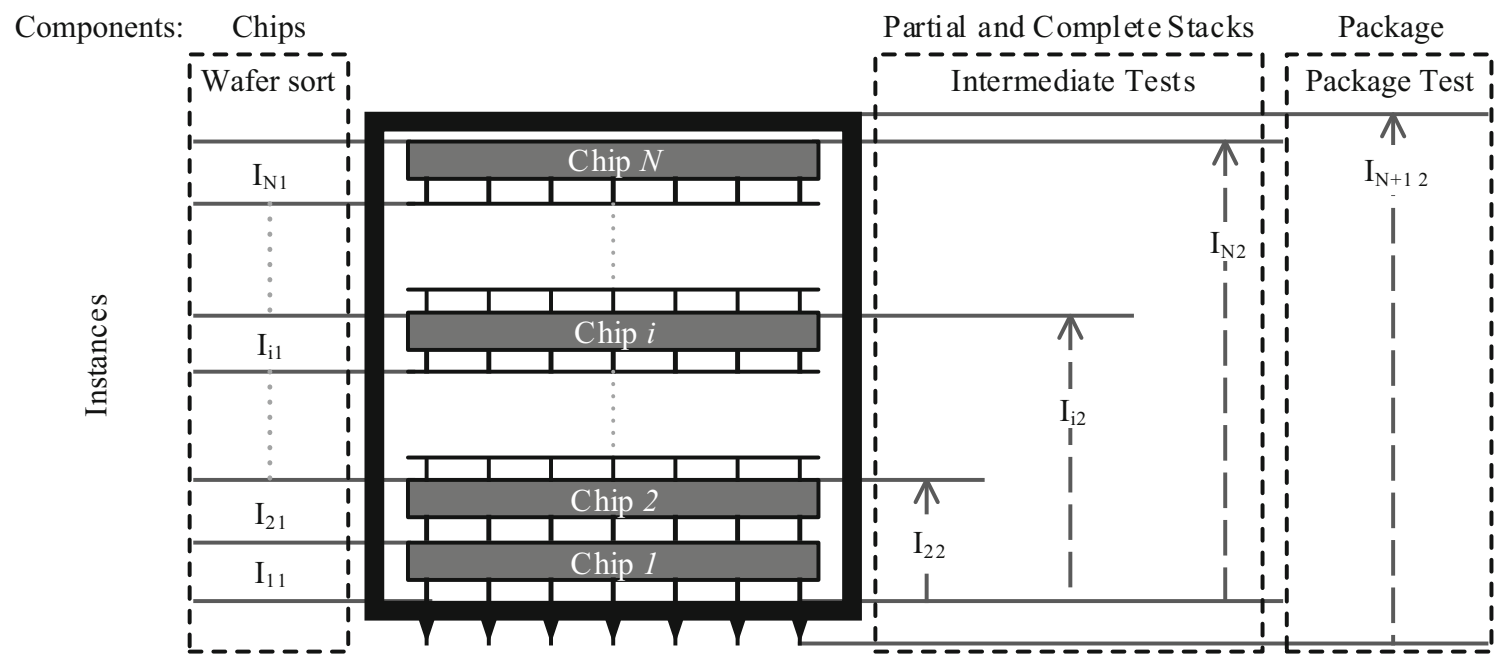

Fig. 4 Components of the 3D Stacked IC that are tested at instance $I_{i j}$. Wafer sort of individual chips are marked on the left, from $I_{11}$ to $I_{N 1}$. Intermediate tests, $I_{22}$ to $I_{N 2}$ of the 3D Stacked IC with 2 to $N$ chips are to the right. Package test $I_{N+12}$ is at the right 


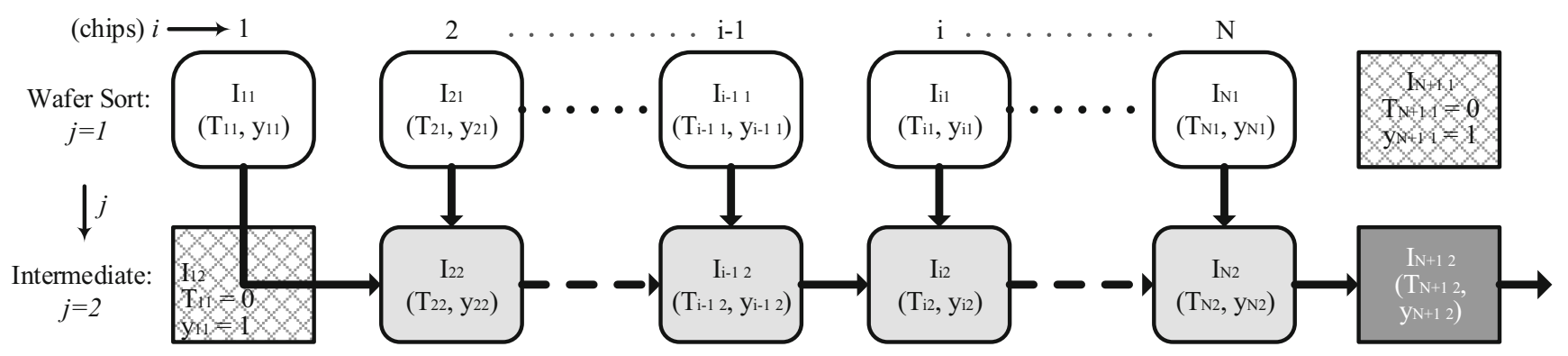

Fig. 5 Test instances $I_{i j}$ of a 3D Stacked IC with $N$ chips in the stack. Upper row indicates wafer sort instances of chips $i=1$ to $N$; from $I_{11}$ to $I_{N 1}$. Lower row indicates intermediate test instances, after stacking chips $i=2$ to $N$ to the incomplete stack, and package test

In the example the yield of chip 2 for case 1 at wafer sort is $y_{21}=0.91$, which means that the effective yield $Y_{21}$, which is wafer sort test of chip 2, is:

$Y_{21}=y_{21}=0.91$

Next, we discuss the effective yield at intermediate and package test instances. Let us consider an arbitrary intermediate or package test instance $I_{i 2}$. For the intermediate test instances the given yield $y_{i 2}$, represents the yield of producing an intermediate stack with $i$ chips, assuming that both components, i.e., the chip $i$ and the intermediate stack with $i-1$ chips, are tested and are defect free. Contrary to wafer sort instances, as seen from Fig. 5, any intermediate test instance, $I_{i 2} \forall i>2$, receives components from the preceding partial stack with $i-1$ and wafer manufacturing stage of chip $i$. Now, if no tests were performed at the wafer sort instance $I_{i 1}$, the defective components will be passed on to the corresponding intermediate test $I_{i 2}$, which would decrease the effective yield at the intermediate test instance $I_{i 2}$. This would result in the effective yield to be the product of the yield of the individual test instances $I_{i 1}$ and $I_{i 2}$, i.e., $\left(y_{i 2} \cdot y_{i 1}\right)$. For example, with the design in Section 4, the yield at the intermediate test instance of stacking chip 1 and chip $2, I_{22}$ is $0.90 \cdot 0.92=0.828$, assuming $X=(1,0),(1),(1)$, as wafer sort has not been performed for chip 2 at instance $I_{21}$. Similarly, the yield at any intermediate instance $I_{i 2}$ depends on tests performed during previous instances $I_{i^{\prime} 2}$, where, $i^{\prime}<i$. Therefore, to calculate the effective yield at any intermediate test instance $I_{i j}$ as a effect of the yield of the instance itself and that of the corresponding wafer sort instance $I_{i 1}$, we use the expression $y_{i j}\left(y_{i 1}^{\bar{x}_{i 1}}\right)$. If $x_{i j}=0$ we get $y_{i 1}^{\bar{x}_{i 1}}=y_{i 1}^{1}=y_{i 1}$ and $x_{i j}=1$ gives $y_{i 1}^{\bar{x}_{i 1}}=y_{i 1}^{0}=1$. Therefore, the expression gives $y_{i j}$ when $x_{i 1}=1$ i.e., when test was performed at wafer sort, and $y_{i j} \cdot y_{i 1}$ when $x_{i 1}=0$ i.e., when no tests were performed at wafer sort. However, the effective yield at any intermediate instance $I_{i 2}$ will also depend on the yield of the preceding intermediate instance $I_{i-12}$ when test was not performed, which in turn will depend on the yield of $I_{i-22}$ instances; $I_{22}$ to $I_{N 2}$. Connecting arrows between instances (boxes) indicate transfer of components. Boxes representing instances $I_{12}$ and $I_{N+11}$ are represented for the sake of convenience

and so on, unless a test had been performed at any of these prior instances. In other words, the yield at any intermediate test instance $I_{i j}$ is a consequence of of the preceding intermediate test instances up to $I_{i^{\prime} j} \quad \forall i^{\prime}<i$, which is the latest intermediate test instance when a test has been performed. Therefore, the first intermediate test instance $I_{22}$ depends only on the preceding wafer sort instances $I_{11}$ and $I_{21}$, as there are no previous intermediate tests possible. Thus we can derive the effective yield at instance $I_{22}$ as:

$Y_{22}=y_{22} \cdot y_{11}^{\bar{x}_{11}} \cdot y_{21}^{\bar{x}_{21}}$

For the 3D Stacked IC in Section 4 with yield Case 1 and test flow $X=(0,1),(1),(1)$, we have $x_{11}=0, x_{21}=1$, $x_{22}=1$ and $x_{32}=1$. Hence we can compute $Y_{22}$ as:

$$
\begin{aligned}
Y_{22} & =y_{22} \cdot y_{11}^{\bar{x}_{11}} \cdot y_{21}^{\bar{x}_{21}} \\
& =0.92 \cdot 0.90^{1} \cdot 0.91^{0} \\
& =0.92 \cdot 0.90 \cdot 1=0.8280
\end{aligned}
$$

Similarly, the effective yield at the following intermediate or package test instance $I_{32}$ depends on all previous tests performed, and can be given as:

$$
\begin{aligned}
Y_{32} & =y_{32} \cdot\left(y_{31}^{\bar{x}_{31}} \cdot\left(y_{22} \cdot\left(y_{11}^{\bar{x}_{11}} \cdot y_{21}^{\bar{x}_{21}}\right)\right)^{\bar{x}_{22}}\right. \\
& =y_{32} \cdot Y_{22}^{\bar{x}_{22}}
\end{aligned}
$$

As seen in Fig. 5, all intermediate test instances, depicted by the bottom row, receive components from preceding wafer sort and intermediate test instances. The yield, depending on the preceding instances, is:

$Y_{i 2}= \begin{cases}y_{i 2} \cdot y_{i 1}^{\bar{x}_{i 1}} \cdot Y_{i-12}^{\bar{x}_{i-12}}, & \text { for } 2<i \leq N+1 \\ y_{22} \cdot y_{11}^{\bar{x}_{11}} \cdot y_{21}^{\bar{x}_{21}}, & \text { for } i=2\end{cases}$

Therefore, in case of package tests, we will have:

$Y_{N+12}=y_{N+12} \cdot y_{N+11}^{\bar{x}_{N+11}} \cdot Y_{N 2}^{\bar{x}_{N 2}}$

Where, it is given that $y_{N+11}=1$, as noted in Table 2 and we set $\bar{x}_{N+11}=1$. 
For the given package test instance $I_{32}$, in this example, the preceding wafer sorts were performed, while the intermediate test instance was avoided; such that $X=$ $(1,1),(0),(1)$. Therefore, the yield of only the intermediate test instance, $I_{22}$, affects the package test instance:

$$
\begin{aligned}
Y_{32} & =y_{32} \cdot y_{31}^{\bar{x}_{31}} \cdot Y_{22}^{\bar{x}_{22}} \\
& =y_{32} \cdot y_{31}^{\bar{x}_{31}} \cdot\left\{y_{22}^{\bar{x}_{22}} \cdot\left(y_{11}^{\bar{x}_{11}} \cdot y_{21}^{\bar{x}_{21}}\right)^{\bar{x}_{22}}\right\} \\
& =0.93 \cdot 1^{1} \cdot\left\{0.92^{1} \cdot\left(0.90^{0} \cdot 0.91^{0}\right)^{1}\right\} \\
& =0.93 \cdot 1 \cdot 0.92 \cdot 1 \cdot 1=0.8556
\end{aligned}
$$

\subsection{Quantity}

At any test instance, the number of units tested is greater than the number of good units obtained, due to imperfect $(<1)$ yield. Therefore, we calculate the expected quantity of good units required at the end of each instance such that a fixed number of good units are obtained from a succeeding manufacturing stage.

Let us start with the package test instance, illustrated by the rightmost box in Fig. 5. With a yield of $y_{N+12}(<$ 1), to obtain $Q_{N+12}=1$ good packages we need to test $Q_{N+12} / y_{N+12}$ packages. Therefore, at the preceding instance $I_{N 2}$, we need to produce $Q_{N 2}=Q_{N+12} / y_{N+12}$ good units. Now, to produce $Q_{N 2}$ good units at the intermediate test instance $I_{N 2}$, we need to test $Q_{N 2} /\left(y_{N 2}\right.$. $\left.y_{N 1}^{\bar{x}_{N 1}}\right)$ units. It is useful to note here that the number of instances that need to be tested during the intermediate test instance $I_{N 2}$ increases by $1 / y_{N 1}$ times if test was not performed at the wafer sort instance $I_{N 1}$. This is due to the share of the defective wafers that pass on to the intermediate stack. Consequently, we need to produce $Q_{N 1}=Q_{N 2} /\left(y_{N 2} \cdot y_{N 1}^{\bar{x}_{N 1}}\right)$ good intermediate stacks and back calculate the number of good units that need to be produced after each test instance up to $Q_{11}$. The quantity of good units required after each test instance $Q_{i j}$ is formulated as follows.

For wafer sort instances:

$Q_{i 1}= \begin{cases}\frac{Q_{i 2}}{\bar{x}_{i+11}}, & 2<i \leq N \\ \frac{y_{i 2} \cdot y_{i+11}}{Q_{22}} & \quad i \leq 2\end{cases}$

For the 3D Stacked IC in Section 4 with yield Case 1 and $X=(0,0),(0),(1)$, we utilize the second part of Eq. 9, since $i=1 \leq 2$. Therefore, the number of units required at the end of instance $I_{11}$ is:

$$
\begin{aligned}
Q_{11} & =\frac{1}{y_{11}^{\bar{x}_{11} \cdot y_{21}^{\bar{x}_{21}} \cdot y_{22}^{\bar{x}_{22}} \cdot y_{32}^{\bar{x}_{32}}}}=1.299 \\
& =\frac{1}{0.90^{1} \cdot 0.91^{0} \cdot 0.92^{1} \cdot 0.93^{1}}=1
\end{aligned}
$$

For intermediate test instances:

$Q_{i 2}= \begin{cases}\frac{Q_{i+12}}{y_{i+12} \cdot y_{i+11}}, & 2 \leq i \leq N-1 \\ \frac{Q_{N+12}}{y_{N+12}}, & i=N\end{cases}$

In the example, the quantity required for package test, when only the wafer sorts have been performed, such that $X=(1,1),(0),(1)$ is:

$Q_{22}=\frac{Q_{32}}{y_{32}}=\frac{1}{0.93}=1.075$

It should be noted that at any intermediate test instance $I_{i 2}$, for each intermediate stack comprising of chips 1 to $i-1$ obtained from instance $I_{i-12}$, per chip $i$ from instance $I_{i 1}$ is stacked. Therefore, we need equal number of units from preceding instances $I_{i 1}$ and $I_{i-12}$, giving $Q_{i 1}=Q_{i-12}$.

\subsection{Time}

An expression to calculate the expected total time taken by any test flow to produce each fault-free packaged 3D Stacked IC is formulated here. The time expected at each test instance depends on the time taken to test each unit at the instance, the effective yield, as well as the number of units required at successive test instances to produce the desired number of good packages. Table 3 is used to list the expected test time at each instance for different test flows required by the 3D Stacked ICs mentioned in Table 1.

The effective test time, $T_{e f f}(i j)$, spent at any instance, $I_{i j}$, depends on the given test time, $T_{i j}$, effective yield, $Y_{i j}$, at the instance and the number of defect-free units that need to be obtained, $Q_{i j}$, and the binary decision variable, $x_{i j}$, is given by:

$T_{e f f}(i j)=\frac{T_{i j}}{Y_{i j}} \cdot Q_{i j} \cdot x_{i j}$

Table 3 Effective test time at each test instance Table 1

\begin{tabular}{lrrrrr}
\hline Test Flow & Teff(11) & Teff(21) & Teff(22) & Teff(32) & \multicolumn{1}{c}{$\tau$} \\
\hline Case 1 & & & & & \\
TA & 12.99 & 12.84 & 35.06 & 75.27 & 136.16 \\
WSPT & 12.99 & 12.84 & 0.00 & 81.81 & 107.64 \\
PT & 0.00 & 0.00 & 0.00 & 99.89 & 99.89 \\
Case 2 & & & & & \\
TA & 27.18 & 26.80 & 57.08 & 95.89 & 206.94 \\
WSPT & 27.18 & 26.80 & 0.00 & 133.18 & 187.16 \\
PT & 0.00 & 0.00 & 0.00 & 267.97 & 267.97 \\
Case 3 & & & & & \\
TA & 72.57 & 71.15 & 108.85 & 132.08 & 384.64 \\
WSPT & 72.57 & 71.15 & 0.00 & 253.99 & 397.71 \\
PT & 0.00 & 0.00 & 0.00 & 996.04 & 996.04 \\
\hline
\end{tabular}


For example, the effective time spent at the intermediate test instance $I_{22}$ when all tests are performed, as seen in the first row of Case 1; such that $X=(1,1),(1),(1)$ is:

$$
\begin{aligned}
T_{\text {eff }}(22) & =\frac{T_{22}}{Y_{22}} \cdot Q_{22} \cdot x_{22} \\
& =\frac{30}{0.92 \cdot 0.90^{0} \cdot 0.91^{0}} \cdot 1.075 \cdot 1=35.06
\end{aligned}
$$

The sum of the effective test times, $T_{e f f}(i j)$, at each instance, $I_{i j}$, gives the expected total test time required, $\tau$, by any selected test flow, $X$, to produce each fault-free packaged 3D Stacked IC as shown below.

$\tau=\sum_{i=1}^{N+1} T_{e f f}(i j) \quad \forall j=1,2$

The expected total test time, assuming a test flow when all instances are tested, as seen in the topmost row of Case 1, gives:

$$
\begin{aligned}
\tau & =T_{\text {eff }}(11)+T_{\text {eff }}(21)+T_{\text {eff }}(22)+T_{\text {eff }}(32) \\
& =12.99+12.84+35.06+75.27=136.16
\end{aligned}
$$

The objective is to find a suitable test flow for any given 3D Stacked IC, such that the expected total test time $\tau$ is minimized.

\section{Test Flow Selection Algorithm (TFSA)}

In this section, we first detail the Test Flow Selection Algorithm (TFSA) and then we detail the computational complexity of the algorithm.

Given the test time $T_{i j}$ and yield $y_{i j}$ at all test instances $I_{i j}$, the TFSA generates a test flow, $X$, by iteratively trying to reduce the expected total test time $\tau$. At each iteration, the test instance that contributes to most reduction in $\tau$ is selected. As discussed in the previous section, we represent a test flow with the vector $X=$ $\left(x_{11} \ldots x_{N 1}\right),\left(x_{22} \ldots x_{N 2}\right),\left(x_{N+12}\right)$, where $\left(x_{N+12}\right)=1$, since package test is always performed.

TFSA, which is detailed in Algorithm 1, in line 1, takes as input $N$ chips where for each test instance $I_{i j}(1 \leq i \leq N$, $1 \leq j \leq 2$ ) the test time $T_{i j}$ and $y_{i j}$ are given. We use the 3D Stacked IC described in Section 4, with yield Case 3 in Table 1 for illustration. In the example, there are 2 chips $(N=2)$; hence, there are $2 \cdot N=4$ possible test instances.

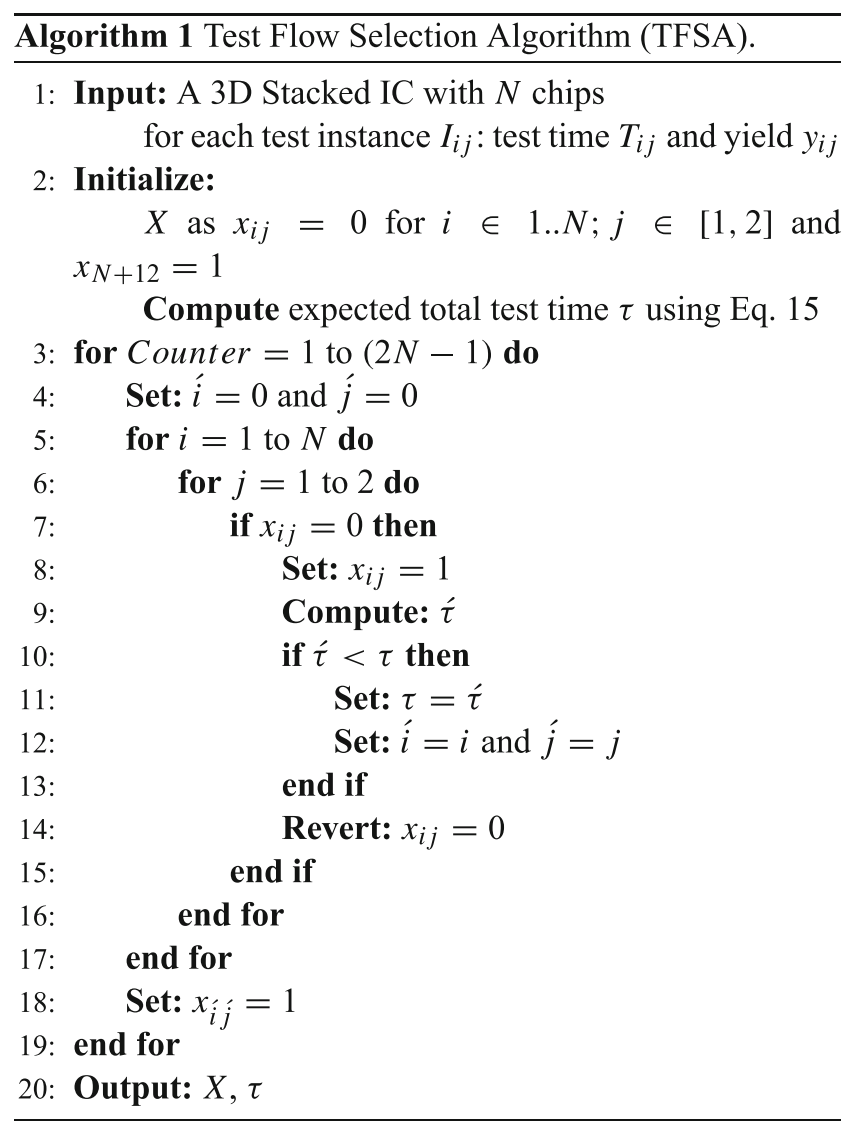

The test flow vector $X$, and the corresponding test cost $\tau$ are initialized in line 2 . All binary decision variables, $x_{i j}$, are initialized such that only package test is applied. For the example, we set $X=(0,0),(0),(1)$. After initialization, the expected time is computed with Eq. 15 to:

$$
\begin{aligned}
\tau & =\sum_{\forall i, j} T_{e f f}(i j) \\
& =T_{e f f}(11)+T_{e f f}(21)+T_{e f f}(22)+T_{e f f}(32) \\
& =0+0+0+\frac{T_{32}}{Y_{32}} \cdot Q_{32} \cdot x_{32} \\
& =\frac{T_{32}}{y_{32} \cdot\left(y_{22} \cdot\left(y_{11}^{\bar{x}_{11}} \cdot y_{21}^{\bar{x}_{21}}\right)\right)^{\bar{x}_{22}}} \cdot 1 \cdot 1 \\
& =\frac{70}{0.53 \cdot\left(0.52 \cdot\left(0.50^{1} \cdot 0.51^{1}\right)\right)^{1}} \cdot 1 \cdot 1
\end{aligned}
$$

As noted in Table 2, $T_{\text {eff }}(12)=0$ and $T_{\text {eff }}(31)=0$.

When only package test is applied, the actual yield at package test takes the yield at all instances into account.

A variable, Counter, is active between lines $3 \rightarrow 19$, to ascertain $2 N-1$ iterations. In this example, Counter iterates from $1 \rightarrow 3$. Variables $i$ and $j$ are reset for the iteration, in line 4.

To scan through all $2 N-1$ test instances $I_{i j}$, variables $i$ and $j$ are defined between lines $5 \rightarrow 17$ and lines $6 \rightarrow 16$, respectively, where $1 \leq i \leq N$ and $1 \leq j \leq 2$. 
During an iteration, each inactive test instance $x_{i j}=0$ is set to $x_{i j}=1$, in line $7 \rightarrow 8$. The corresponding test cost $\dot{\tau}$ is computed in line 9, as a result of the modified test flow, to evaluate if there is a benefit to include the test instance in the test flow. In the first iteration, the matrix is updated to $(1,0),(0),(1)$.

At line 9, the effective total test time $\tau$ for the current test flow $X=(1,0),(0),(1)$ is computed as:

$$
\begin{aligned}
\tau= & \sum_{\forall i, j} T_{e f f}(i j) \\
= & T_{e f f}(11)+T_{e f f}(21)+T_{e f f}(22)+T_{e f f}(32) \\
= & \frac{T_{11}}{Y_{11}} \cdot Q_{11} \cdot x_{11}+0+0+\frac{T_{32}}{Y_{32}} \cdot Q_{32} \cdot x_{32} \\
= & \frac{T_{11}}{y_{11}} \cdot \frac{1}{y_{12} \cdot y_{22} \cdot y_{32}} \cdot 1+0+0 \\
& +\frac{T_{32}}{y_{32} \cdot\left(y_{22} \cdot\left(y_{11}^{\bar{x}_{11}} \cdot y_{21}^{\bar{x}_{21}}\right)\right)^{\bar{x}_{22}}} \cdot 1 \cdot 1 \\
= & \frac{10}{0.50} \cdot \frac{1}{0.51 \cdot 0.52 \cdot 0.53} \cdot 1 \\
& +\frac{70}{0.53 \cdot\left(0.52 \cdot\left(0.50^{0} \cdot 0.51^{1}\right)\right)^{1}} \cdot 1 \cdot 1
\end{aligned}
$$

Note, in this case, wafer sort is applied to chip 1, which means $y_{11}$ is used at test instance $I_{11}$, and not in test instance $I_{32}$.

If the new test cost $\dot{\tau}$ is lower than all previously computed test costs $\tau$ (line 10), the test cost is updated as $\tau=\hat{\tau}$ and the indices $i$ and $j$ are recorded, at lines 11 and 12 , respectively. In the example, $\tau=640$ and $\tau=996$. Hence, a better solution is found, thus replacing $\tau$ by $\dot{\tau}$. The algorithm continues with the test flow $(0,1),(0),(1)$ which gives $\dot{\tau}=640$, same as the present value of $\tau=$

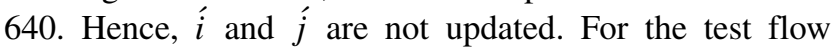
$(0,0)$, (1), (1), we get $\tau=558<\tau=640$, and update indices $\hat{i}^{\prime}=2$ and $j^{\prime}=2$.

Hence, at the end of the first iteration, in line 18, we update the test flow to $X=(0,0),(1),(1)$.

Similarly, at the end of the second iteration Counter $=$ 2, we will have $X=(1,0),(1),(1)$ and $\tau=487$.
Eventually, at the third and final iteration Counter $=2 \mathrm{~N}-$ $1=3$, we will have $X=(1,1),(1),(1)$ and $\tau=384$. Thus, for this example, $(1,1),(1),(1)$, means wafer sort is applied to both chip 1 and chip 2, intermediate test is applied to the stack of chips $1+2$ and package test is applied to the complete stack. The expected total test time is 384 .

\subsection{Complexity Estimation}

There are two nested iterations in algorithm 1. The outer iteration, for loops between lines $3 \rightarrow 19$, iterates Counter from 1 to $2 N-1$, and the inner iteration, for loops between lines $5 \rightarrow 17$, iterates $i$ from 1 to $N$. For each $i, j$ takes two values 1 and 2 . Thus, the complexity is the product of the number of iterations of each loop, i.e., $(2 N-1) \cdot N \cdot 2=$ $4 N^{2}-2 N$, which is of order $O\left(N^{2}\right)$.

\section{Experiments}

In this section we present two sets of experiments. First we compare the expected total test times obtained from TFSA with respect to three straightforward test flows and the test flow obtained by exhaustive search. Next, the TFSA is integrated with test planning of core-based 3D Stacked ICs with a IEEE 1500 based test architecture, to optimize the test time.

\subsection{Test Flow Selection}

The objective is to compare the expected total test time by applying TFSA and that required with the three straightforward test flow schemes (TA, WSPT, and PT) as well as against exhaustive search.

Experiments were performed on two sets of 3D Stacked IC designs with 2 to 10 chips in the stack. The 3D Stacked IC designs are detailed in Table 5. For example, in case of both Set 1 and Set 2, SIC 2 consists of three chips in the stack, chip 1, 2, and 3. The test time and yield values of each chip in the 3D Stacked IC designs at wafer sort are given for Set 1 and Set 2 in Table 4. In Set 1, chip 1 has a test time of 1000 time units and a yield of 0.62 . The test times
Table 4 Test times and yields of Chips 1 to 10

\begin{tabular}{lllllllllll}
\hline Chip & 1 & 2 & 3 & 4 & 5 & 6 & 7 & 8 & 9 & 10 \\
\hline Set 1 & & & & & & & & & \\
$\quad$ Test time & 1000 & 1000 & 1000 & 1000 & 1000 & 1000 & 1000 & 1000 & 1000 & 1000 \\
$\quad$ Yield & 0.62 & 0.66 & 0.70 & 0.74 & 0.78 & 0.82 & 0.86 & 0.90 & 0.94 & 0.98 \\
Set 2 & & & & & & & & & & \\
$\quad$ Test time & 1000 & 1000 & 1000 & 1000 & 1000 & 1000 & 1000 & 1000 & 1000 & 1000 \\
Yield & 0.98 & 0.94 & 0.90 & 0.86 & 0.82 & 0.78 & 0.74 & 0.70 & 0.66 & 0.62 \\
\hline
\end{tabular}


Table 5 Designs

\begin{tabular}{ll}
\hline $\begin{array}{l}\text { 3D Stacked IC } \\
\text { designs }\end{array}$ & $\begin{array}{l}\text { Chips in the 3D Stacked ICs as detailed } \\
\text { in Table 4. First chip is lowermost. }\end{array}$ \\
\hline$S I C_{1}$ & 1,2 \\
$S I C_{2}$ & $1,2,3$ \\
$S I C_{3}$ & $1,2,3,4$ \\
$S I C_{4}$ & $1,2,3,4,5$ \\
$S I C_{5}$ & $1,2,3,4,5,6$ \\
$S I C_{6}$ & $1,2,3,4,5,6,7$ \\
$S I C_{7}$ & $1,2,3,4,5,6,7,8$ \\
$S I C_{8}$ & $1,2,3,4,5,6,7,8,9$ \\
$S I C_{9}$ & $1,2,3,4,5,6,7,8,9,10$ \\
\hline
\end{tabular}

are kept constant for all chips in the stack for both Set 1 and Set 2, to emphasize the difference among the expected total test times. However, the yield values for Set 1 and Set 2 are changed to emphasize the differences among the test flows obtained. For intermediate tests and package test, we assume an additional test time of 1000 units and the given yield at the test instance to be 0.70 . For instance, the test time assumed during the package test of $S I C_{3}$ is the sum of the the test times of each individual chip - chips 1,2 and 3 -, two layers of interconnects - between chips 1 and 2, and chips 2 and $3-$ give an additional $2 \times 1000$, and finally 1000 time units for testing the package itself (Table 5).
The results of the comparison between TFSA, TA, PT, WSPT and exhaustive search are collated in Table 6. Table 6 is organized as follows. The leftmost column lists the 3D Stacked IC designs. The following group of five columns list the expected total test times required by each method. The rightmost group of four columns depicts for each method the overhead in expected total test time time compared to the optimal expected total test time obtained by exhaustive search. The most significant points that can be drawn from Table 6 are:

- TFSA generates test flows and corresponding test times very close to exhaustive search in most cases.

- PT has a low expected total test time for 3D Stacked ICs with up to three chips in the stack: for $S I C_{1}$ of Set 1 , the result is only $1 \%$ away from optimum, whereas the optimal is obtained for $S I C_{1}$ and $S I C_{2}$ of Set 2. However, for all other cases, PT produces results that are far from optimum. As the number of chips in the 3D Stacked IC increases, the performance of PT deteriorates.

- TA results give expected total test times that are about $40 \%$ more than optimum for Set 1 and over $80 \%$ worse at an average for Set 2 .

- WSPT is not as efficient as the TFSA. However, it is interesting to note that WSPT produces optimal results when the number of chips is less than 4 , and WSPT is
Table 6 Comparison of expected total test times

\begin{tabular}{|c|c|c|c|c|c|c|c|c|c|}
\hline \multirow{2}{*}{$\begin{array}{l}\text { 3D Stacked } \\
\text { ICs }\end{array}$} & \multicolumn{5}{|c|}{ Total test time $(\tau)$} & \multicolumn{4}{|c|}{ Difference $(\%)$} \\
\hline & Exhaustive & TFSA & TA & PT & WSPT & TFSA & TA & PT & WSPT \\
\hline \multicolumn{10}{|l|}{ Set 1} \\
\hline$S I C_{1}$ & 10874 & 10874 & 13812 & 10972 & 10874 & 0 & 27 & 1 & 0 \\
\hline $\mathrm{SIC}_{2}$ & 21656 & 21656 & 29402 & 33588 & 21656 & 0 & 36 & 55 & 0 \\
\hline $\mathrm{SIC}_{3}$ & 38277 & 38277 & 53088 & 86456 & 38277 & 0 & 39 & 126 & 0 \\
\hline $\mathrm{SIC}_{4}$ & 63842 & 63842 & 88354 & 197930 & 63842 & 0 & 38 & 210 & 0 \\
\hline$S_{I} C_{5}$ & 101631 & 101631 & 140179 & 413792 & 103042 & 0 & 36 & 302 & 1 \\
\hline $\mathrm{SIC}_{6}$ & 159216 & 178426 & 215669 & 801922 & 162933 & 12 & 35 & 404 & 2 \\
\hline $\mathrm{SIC}_{7}$ & 184017 & 184017 & 324978 & 1454734 & 254110 & 0 & 77 & 691 & 38 \\
\hline$S I C_{8}$ & 346572 & 346572 & 482610 & 2487197 & 392444 & 0 & 39 & 618 & 13 \\
\hline$S I C_{9}$ & 503730 & 503730 & 709280 & 4028502 & 601658 & 0 & 41 & 700 & 19 \\
\hline \multicolumn{10}{|l|}{ Set 2} \\
\hline$S I C_{1}$ & 4874 & 4874 & 11682 & 4874 & 8743 & 0 & 140 & 0 & 79 \\
\hline$S I C_{2}$ & 11604 & 11604 & 25711 & 11604 & 17965 & 0 & 122 & 0 & 55 \\
\hline $\mathrm{SIC}_{3}$ & 25702 & 25702 & 47430 & 25702 & 32619 & 0 & 85 & 10 & 27 \\
\hline $\mathrm{SIC}_{4}$ & 45946 & 45946 & 80144 & 55971 & 55632 & 0 & 74 & 22 & 21 \\
\hline$S I C_{5}$ & 75558 & 75558 & 128577 & 123013 & 91441 & 0 & 70 & 63 & 21 \\
\hline$S I C_{6}$ & 121359 & 127854 & 199481 & 277057 & 146744 & 5 & 64 & 128 & 21 \\
\hline $\mathrm{SIC}_{7}$ & 188408 & 188408 & 302500 & 646197 & 231631 & 0 & 61 & 243 & 23 \\
\hline$S I C_{8}$ & 284257 & 284257 & 451419 & 1573533 & 361253 & 0 & 59 & 454 & 27 \\
\hline$S I C_{9}$ & 421316 & 421316 & 665931 & 4028502 & 558309 & 0 & 58 & 856 & 33 \\
\hline
\end{tabular}


only a few $\%$ away from optimum when the number of chips in the stack is less than eight.

Table 7 lists the test flows obtained by the exhaustive search and the TFSA, respectively. It is interesting to note, first of all, the test flows proposed by the TFSA are, in most cases, the same as the test flows obtained from exhaustive search. However, for exhaustive search the expected total test time for $2^{2 N-1}$ test flows need to be evaluated, whereas TFSA only compares $(2 N-1)^{2}$ test flows. Therefore, TFSA requires lower computation time, as compared to exhaustive search, to determine a test flow for 3D Stacked ICs with more than 2 chips in the stack. In case of 3D Stacked ICs with 9 chips in the stack, for TFSA, the test flow was determined in just over 2 minutes, whereas exhaustive search required longer than 2 days to arrive at the same result in Table 7. Secondly, the optimal test flow does not follow a regular pattern. For example, $S I C_{6}$ and $S I C_{7}$ of Set 1 differ by only one chip. But the test flows are very different. In addition, it is also observed that performing wafer sort pays off in most cases.

\subsection{Test Architecture Design}

In the second set of experiments, the goal is to compare the expected total test times obtained by integrating test architecture design and test planning schemes with different test flows. We evaluate (1) TFSA against three straightforward test flow schemes (TA, WSPT, and PT) against an exhaustive search of all possible test flows, and (2) the test flows on three test architecture designs and test planning schemes. The objective here is to integrate test flow selection and test architecture design to obtain the minimal test cost.

We assume that the given 3D Stacked ICs are core-based, and each core is provided with a IEEE 1500 based core test wrapper. The problem at system-level is given a TAM width (W) to find the most suitable number of TAM groups, their widths, and assign the cores to the TAM groups such that test time is minimized. The three test planning schemes that we assume are:

- Scheme 1, the TAM for each chip is optimized independently of all other chips in the 3D Stacked IC. It means that each chip gets the TAM that is most suitable for its wafer sort. Note that after the optimization additional TAM wires can be added to a chip. For example, if the top chip requires a wide TAM while all other chips only need a narrow TAM, the wide TAM is added to all chips to make testing of the top chip possible at package test.

- Scheme 2, the TAM for the lowest chip is optimized and that TAM architecture is used for all chips in the 3D Stacked IC. In this case, all chips use the TAM

Table 7 Comparison of test flows obtained with exhaustive search against TFSA

\begin{tabular}{|c|c|c|}
\hline Design & Test flow for exhaustive search & Test flow for TFSA \\
\hline \multicolumn{3}{|l|}{ Set 1} \\
\hline$S I C_{1}$ & $(1,1),(0),(1)$ & $(1,1),(0),(1)$ \\
\hline$S I C_{2}$ & $(1,1,1),(0,0),(1)$ & $(1,1,1),(0,0),(1)$ \\
\hline$S_{I C} C_{3}$ & $(1,1,1,1),(0,0,0),(1)$ & $(1,1,1,1),(0,0,0),(1)$ \\
\hline $\mathrm{SIC}_{4}$ & $(1,1,1,1,1),(0,0,0,0),(1)$ & $(1,1,1,1,1),(0,0,0,0),(1)$ \\
\hline$S I C_{5}$ & $(1,1,1,1,1,1),(0,1,0,0,0),(1)$ & $(1,1,1,1,1,1),(0,1,0,0,0),(1)$ \\
\hline$S I C_{6}$ & $(0,0,1,1,1,1,1),(1,0,1,0,0,0),(1)$ & $(1,1,1,1,1,1,1),(0,1,0,0,0,0),(1)$ \\
\hline$S I C_{7}$ & $(1,1,1,1,1,1,1,1),(0,0,0,0,0,1,0),(1)$ & $(1,1,1,1,1,1,1,1),(0,1,0,0,0,0,0),(1)$ \\
\hline$S I C_{8}$ & $(1,1,1,1,1,1,1,1,1),(0,1,0,0,1,0,0,0),(1)$ & $(1,1,1,1,1,1,1,1,1),(0,1,0,0,1,0,0,0),(1)$ \\
\hline$S I C_{9}$ & $(1,1,1,1,1,1,1,1,1,1),(0,1,0,0,1,0,0,0,0),(1)$ & $(1,1,1,1,1,1,1,1,1,1),(0,1,0,0,1,0,0,0,0),(1)$ \\
\hline \multicolumn{3}{|l|}{ Set 2} \\
\hline$S I C_{1}$ & $(0,0),(0),(1)$ & $(0,0),(0),(1)$ \\
\hline$S I C_{2}$ & $(0,0,0),(0,0),(1)$ & $(0,0,0),(0,0),(1)$ \\
\hline$S I C_{3}$ & $(0,0,1,1),(1,0,0),(1)$ & $(0,0,1,1),(0,1,0),(1)$ \\
\hline $\mathrm{SIC}_{4}$ & $(0,0,0,1,1),(0,0,1,0),(1)$ & $(0,0,0,1,1),(0,0,1,0),(1)$ \\
\hline$S I C_{5}$ & $(0,0,0,1,1,1),(0,0,1,1,0),(1)$ & $(0,0,0,1,1,1),(0,0,1,1,0),(1)$ \\
\hline$S I C_{6}$ & $(0,0,1,1,1,1,1),(0,0,1,1,0,0),(1)$ & $(0,0,0,1,1,1,1),(0,0,1,1,0,0),(1)$ \\
\hline $\mathrm{SIC}_{7}$ & $(0,0,0,1,1,1,1,1),(0,0,1,1,1,0,0),(1)$ & $(0,0,0,1,1,1,1,1),(0,0,1,1,1,0,0),(1)$ \\
\hline$S I C_{8}$ & $(0,0,0,1,1,1,1,1,1),(0,0,1,1,1,0,0,0),(1)$ & $(0,0,0,1,1,1,1,1,1),(0,0,1,1,1,0,0,0),(1)$ \\
\hline$S I C_{9}$ & $(0,0,0,1,1,1,1,1,1,1),(0,0,1,1,1,0,0,0,0),(1)$ & $(0,0,0,1,1,1,1,1,1,1),(0,0,1,1,1,0,0,0,0),(1)$ \\
\hline
\end{tabular}


Table 8 Experimental data

\begin{tabular}{lllll}
\hline Label & Design & Cores & Time & Yield \\
\hline $\mathrm{D}$ & $\mathrm{d} 695$ & 11 & 695828 & 0.65 \\
$\mathrm{G}$ & $\mathrm{g} 1023$ & 15 & 731423 & 0.65 \\
$\mathrm{P}$ & $\mathrm{p} 34392$ & 20 & 16372887 & 0.75 \\
$\mathrm{~T}$ & $\mathrm{t} 512505$ & 32 & 165324037 & 0.75 \\
Intermediate test & & & 10000 & 0.65 \\
Package test & & & 10000 & 0.75 \\
\hline
\end{tabular}

optimized for wafer sort test of the lowest chip in the 3D Stacked IC.

- Scheme SIC, ILP is used to optimize the test architecture for a given test flow. Our ILP scheme [12] is extended from only accepting WSPT to allow an arbitrary test flow.

The four ITC' 02 benchmarks used to construct the corebased 3D Stacked ICs are: d695 (D), g1023 (G), p34392 (P), and t512505 (T), as detailed in Table 8. To give an indication of the complexity of the designs, Table 8 details the number of cores in the third column. The test time for each design, when all scan chains within the design are concatenated to a single wrapper chain i.e. the TAM width is 1 , is tabulated in the fourth column. The yield for each design, as assumed for experiments in this paper, are shown in the last column. For example, d695, represented as D, contains 11 cores, requires a test time of 695828 clock cycles and has a yield of 0.65 . The two bottom rows indicate the additional test time incurred during each intermediate test, and during the package test respectively. At each intermediate test an additional 10000 time units are added to the total time at the test instance. For instance, the test time assumed during the package test of DGPT is the sum of the test times of each chip - D, G, P and T - three layers of interconnects in between correspond to $3 \times 10000$, and finally 10000 for the package itself. The yield at intermediate test is set to 0.65 and the yield at package test is set to 0.75 .

Each ITC' 02 benchmark in Table 8 represents a chip in a 3D Stacked IC. By combining the four benchmarks in various ways, we constructed 3D Stacked ICs with 2, 3 and 4 chips. In total we created 9 designs (DP, DT, GP, GT, DGP, DGT, DPT, GPT, and DGPT) where for example the DP design is a 3D Stacked IC with 2 chips consisting of d695 and p34392 where d695 is the lowest chip. The test time required to test each unit at any test instance is obtained from test architecture design and test planning using either Scheme 1, Scheme 2 or Scheme SIC, detailed in [12]. Note that the test time obtained from test architecture design and test planning of the 3D Stacked IC does not change with yield or the output quantity [12].

In the experiments, for each design we applied the four test flows and at each test flow we used the three test architecture design schemes. The results are collated in Table 9, which is organized as follows. There is a subtable for each of the 9 designs (DP, DT, GP, GT, DGP, DGT, DPT, GPT, and DGPT). Each sub-table is organized in the same manner. As an example, we take the sub-table for DP at the upper left corner of Table 9. The four test flows are listed to the left and for each test flow the test time using the three test architecture schemes are reported. For example, the test time using Scheme SIC with a test flow obtained with TFSA is 5326513. Each test time is compared in two ways. First, at a given test flow the test times are compared against Scheme SIC. For example, the test time obtained with Scheme SIC is $10 \%$ lower than that of Scheme 1, where each test architecture scheme is applied on the corresponding test flow obtained with TFSA. Second, for a given test architecture the test times at different test flows are compared. For example, with the SIC Scheme, PT requires 56\% higher test time as compared to TFSA.

The results indicate that Scheme SIC is best for all cases. In some cases, Scheme SIC versus Scheme 2 on design GP is $9 \%$ better, but in some cases, for example DGPT Scheme SIC is $56 \%$ better than Scheme 2, with each test architecture scheme using the test flow obtained by TFSA.

The results indicate that WSPT is as good as TFSA in many cases; however, overall TFSA is close to exhaustive search. In Table 10 the test flows with the lowest test times on any test architecture scheme produced by TFSA is compared against exhaustive search on the designs. For example, the test flow for design DP using exhaustive search is $X=(1,1),(0),(1)$, which means wafer sort of D and P and package test of DP (for details, refer to Section 5). 
Table 9 Expected total test times required by 3D stacked ICs for different test flow and test architecture schemes

\begin{tabular}{|c|c|c|c|c|c|c|c|c|c|c|c|}
\hline \multicolumn{6}{|c|}{ different test flow and test architecture schemes } & & \multirow{2}{*}{\multicolumn{3}{|c|}{ Test architecture scheme }} & \multirow{2}{*}{\multicolumn{2}{|c|}{ vs SIC (\%) }} \\
\hline & & & & & & \multirow{3}{*}{$\begin{array}{l}\text { Test } \\
\text { flow }\end{array}$} & & & & & \\
\hline \multirow{3}{*}{$\begin{array}{l}\text { Test } \\
\text { flow }\end{array}$} & \multicolumn{3}{|c|}{ Test architecture scheme } & \multicolumn{2}{|c|}{ vs $\operatorname{SIC}(\%)$} & & \multirow{2}{*}{$\begin{array}{l}\text { Scheme } \\
\text { SIC }\end{array}$} & \multirow{2}{*}{$\begin{array}{l}\text { Scheme } \\
1\end{array}$} & \multirow{2}{*}{$\begin{array}{l}\text { Scheme } \\
2\end{array}$} & \multirow{2}{*}{$\begin{array}{l}\text { Scheme } \\
1\end{array}$} & \multirow{2}{*}{$\begin{array}{l}\text { Scheme } \\
2\end{array}$} \\
\hline & Scheme & Scheme & Scheme & Scheme & Scheme & & & & & & \\
\hline & SIC & 1 & 2 & 1 & 2 & & \multicolumn{5}{|c|}{ vs TFSA $(\%)$} \\
\hline & \multicolumn{5}{|c|}{ SIC: DP; TAM width $=10$} & WSPT & $\begin{array}{l}\text { VS TFSA } \\
10\end{array}$ & $\begin{array}{l}\%) \\
12\end{array}$ & 12 & & \\
\hline TFSA & $5.3 \mathrm{E}+6$ & $5.9 \mathrm{E}+6$ & $6.7 \mathrm{E}+6$ & 11 & 14 & $\mathrm{TA}$ & 25 & 31 & 32 & & \\
\hline WSPT & $5.3 \mathrm{E}+6$ & $5.9 \mathrm{E}+6$ & $6.7 \mathrm{E}+6$ & 11 & 14 & PT & 12 & 16 & 17 & & \\
\hline $\mathrm{TA}$ & $1.0 \mathrm{E}+7$ & $1.2 \mathrm{E}+7$ & $1.4 \mathrm{E}+7$ & 20 & 17 & & \multicolumn{5}{|c|}{ SIC: DGT; TAM width $=25$} \\
\hline \multirow[t]{2}{*}{ PT } & $8.3 \mathrm{E}+6$ & $9.5 \mathrm{E}+6$ & $1.1 \mathrm{E}+7$ & 14 & 16 & TFSA & $3.7 \mathrm{E}+7$ & $4.1 \mathrm{E}+7$ & $4.7 \mathrm{E}+7$ & 11 & 15 \\
\hline & \multicolumn{5}{|c|}{ vs TFSA $(\%)$} & WSPT & $4.1 \mathrm{E}+7$ & $4.6 \mathrm{E}+7$ & $5.3 \mathrm{E}+7$ & 12 & 15 \\
\hline WSPT & 0 & 0 & 0 & & & $\mathrm{TA}$ & $4.7 \mathrm{E}+7$ & $5.4 \mathrm{E}+7$ & $6.2 \mathrm{E}+7$ & 15 & 15 \\
\hline $\mathrm{TA}$ & 94 & 102 & 104 & & & PT & $4.2 \mathrm{E}+7$ & $4.8 \mathrm{E}+7$ & $5.6 \mathrm{E}+7$ & 14 & 17 \\
\hline \multirow[t]{2}{*}{ PT } & 56 & 62 & 65 & & & & \multicolumn{5}{|c|}{ vs TFSA $(\%)$} \\
\hline & \multicolumn{5}{|c|}{ SIC: DT; TAM width $=16$} & WSPT & 12 & 14 & 15 & & \\
\hline TFSA & $3.0 \mathrm{E}+7$ & $3.7 \mathrm{E}+7$ & $4.5 \mathrm{E}+7$ & 23 & 22 & $\mathrm{TA}$ & 27 & 33 & 34 & & \\
\hline WSPT & $3.0 \mathrm{E}+7$ & $3.7 \mathrm{E}+7$ & $4.5 \mathrm{E}+7$ & 23 & 22 & PT & 14 & 18 & 20 & & \\
\hline TA & $6.2 \mathrm{E}+7$ & $7.6 \mathrm{E}+7$ & $9.6 \mathrm{E}+7$ & 23 & 26 & & \multicolumn{5}{|c|}{ SIC: DPT; TAM width $=30$} \\
\hline PT & $5.0 \mathrm{E}+7$ & $5.9 \mathrm{E}+7$ & $7.2 \mathrm{E}+7$ & 18 & 22 & TFSA & $3.5 \mathrm{E}+7$ & $3.8 \mathrm{E}+7$ & $4.4 \mathrm{E}+7$ & 9 & 16 \\
\hline & vs TFSA & & & & & WSPT & $3.7 \mathrm{E}+7$ & $4.2 \mathrm{E}+7$ & $4.9 \mathrm{E}+7$ & 14 & 17 \\
\hline WSPT & 0 & 0 & 0 & & & $\mathrm{TA}$ & $4.4 \mathrm{E}+7$ & $5.1 \mathrm{E}+7$ & $5.9 \mathrm{E}+7$ & 16 & 16 \\
\hline TA & 106 & 105 & 111 & & & PT & $3.8 \mathrm{E}+7$ & $4.3 \mathrm{E}+7$ & $5.1 \mathrm{E}+7$ & 13 & 19 \\
\hline PT & 66 & 60 & 57 & & & & vs TFSA & $\%)$ & & & \\
\hline & SIC: GP; & [AM widtl & $=22$ & & & WSPT & 7 & 9 & 10 & & \\
\hline TFSA & $2.5 \mathrm{E}+6$ & $2.8 \mathrm{E}+6$ & $3.1 \mathrm{E}+6$ & 12 & 11 & $\mathrm{TA}$ & 25 & 30 & 33 & & \\
\hline WSPT & $2.5 \mathrm{E}+6$ & $2.8 \mathrm{E}+6$ & $3.1 \mathrm{E}+6$ & 12 & 11 & $\mathrm{PT}$ & 9 & 13 & 15 & & \\
\hline $\mathrm{TA}$ & $4.7 \mathrm{E}+6$ & $5.3 \mathrm{E}+6$ & $5.6 \mathrm{E}+6$ & 13 & 6 & & SIC: GPT & TAM wid & $h=16$ & & \\
\hline PT & $3.8 \mathrm{E}+6$ & $4.4 \mathrm{E}+6$ & $4.9 \mathrm{E}+6$ & 16 & 11 & TFSA & $6.5 \mathrm{E}+7$ & $7.2 \mathrm{E}+7$ & $8.3 \mathrm{E}+7$ & 11 & 15 \\
\hline & vs TFSA & & & & & WSPT & $7.0 \mathrm{E}+7$ & $7.9 \mathrm{E}+7$ & $9.1 \mathrm{E}+7$ & 13 & 15 \\
\hline WSPT & 0 & 0 & 0 & & & $\mathrm{TA}$ & $8.2 \mathrm{E}+7$ & $9.5 \mathrm{E}+7$ & $1.1 \mathrm{E}+8$ & 16 & 16 \\
\hline $\mathrm{TA}$ & 92 & 88 & 82 & & & PT & $7.1 \mathrm{E}+7$ & $8.1 \mathrm{E}+7$ & $9.5 \mathrm{E}+7$ & 14 & 17 \\
\hline PT & 56 & 56 & 60 & & & & vs TFSA & $\%)$ & & & \\
\hline & SIC: GT; & [AM widtl & $=22$ & & & WSPT & 7 & 9 & 10 & & \\
\hline TFSA & $2.2 \mathrm{E}+7$ & $2.6 \mathrm{E}+7$ & $3.2 \mathrm{E}+7$ & 18 & 23 & $\mathrm{TA}$ & 26 & 32 & 33 & & \\
\hline WSPT & $2.2 \mathrm{E}+7$ & $2.6 \mathrm{E}+7$ & $3.2 \mathrm{E}+7$ & 18 & 23 & PT & 9 & 13 & 15 & & \\
\hline $\mathrm{TA}$ & $4.5 \mathrm{E}+7$ & $5.1 \mathrm{E}+7$ & $5.6 \mathrm{E}+7$ & 13 & 10 & & SIC: DGF & T; TAM w & $\mathrm{dth}=30$ & & \\
\hline PT & $3.6 \mathrm{E}+7$ & $4.2 \mathrm{E}+7$ & $5.2 \mathrm{E}+7$ & 17 & 24 & TFSA & $3.5 \mathrm{E}+7$ & $4.3 \mathrm{E}+7$ & $6.3 \mathrm{E}+7$ & 23 & 47 \\
\hline & vs TFSA & & & & & WSPT & $5.0 \mathrm{E}+7$ & $6.4 \mathrm{E}+7$ & $9.9 \mathrm{E}+7$ & 28 & 55 \\
\hline WSPT & 0 & 0 & 0 & & & $\mathrm{TA}$ & $5.2 \mathrm{E}+7$ & $6.2 \mathrm{E}+7$ & $9.2 \mathrm{E}+7$ & 19 & 48 \\
\hline $\mathrm{TA}$ & 106 & 94 & 76 & & & $\mathrm{PT}$ & $6.8 \mathrm{E}+7$ & $9.0 \mathrm{E}+7$ & $1.3 \mathrm{E}+8$ & 32 & 44 \\
\hline PT & 65 & 61 & 64 & & & & vs TFSA & $\%)$ & & & \\
\hline & SIC: DGl & ;AM wic & $h=25$ & & & WSPT & 45 & 48 & 57 & & \\
\hline TFSA & $4.1 \mathrm{E}+6$ & $4.5 \mathrm{E}+6$ & $5.2 \mathrm{E}+6$ & 10 & 16 & TA & 45 & 45 & 45 & & \\
\hline WSPT & $4.4 \mathrm{E}+6$ & $5.0 \mathrm{E}+6$ & $5.8 \mathrm{E}+6$ & 14 & 16 & PT & 97 & 108 & 110 & & \\
\hline
\end{tabular}

Table 9 (continued) 
Table 10 Test flow used with SIC Scheme to minimize test time

\begin{tabular}{lll}
\hline Design & Exhaustive & TFSA \\
\hline DP & $(1,1),(0),(1)$ & $(1,1),(0),(1)$ \\
DT & $(1,1),(0),(1)$ & $(1,1),(0),(1)$ \\
GP & $(1,1),(0),(1)$ & $(1,1),(0),(1)$ \\
GT & $(1,1),(0),(1)$ & $(1,1),(0),(1)$ \\
DGP & $(1,1,0),(1,0),(1)$ & $(1,1,0),(1,0),(1)$ \\
DGT & $(1,1,0),(1,0),(1)$ & $(1,1,0),(1,0),(1)$ \\
DPT & $(1,1,0),(1,0),(1)$ & $(1,1,0),(1,0),(1)$ \\
GPT & $(1,1,0),(1,0),(1)$ & $(1,1,0),(1,0),(1)$ \\
DGPT & $(1,1,1,0),(1,1,0),(1)$ & $(1,1,1,0),(1,1,0),(1)$ \\
\hline
\end{tabular}

\section{Conclusion}

In this paper, we illustrate the importance of test flow selection to reduce the expected total test time to produce each 3D Stacked IC. We propose a test flow selection algorithm (TFSA) to find the most suitable test flow for a given 3D Stacked IC. We evaluated the test flow obtained from TFSA, against three straightforward test flows and that obtained by exhaustive search. In the experiments we also compare the different test flows after integrating each with three test architecture design and test planning schemes. The experimental results demonstrate the importance to have methods both to find the test flow as well as test architecture design and test planning. It is observed that TFSA provides the optimal test flow, identical to exhaustive search, for all benchmarks. The test time can be further reduced by using both test architecture optimization and TFSA. For the benchmarks used in this paper, straightforward test flows like WSPT may perform as good as TFSA.

1) TFSA generates test flows and the corresponding test times very close to exhaustive search;

2) TFSA and a 3D Stacked IC optimized test architecture performs best with respect to test time, and

3) WSPT provides the minimum test time among the three straightforward test flow schemes and in many cases is equal to that with TFSA.

Open Access This article is distributed under the terms of the Creative Commons Attribution 4.0 International License (http:// creativecommons.org/licenses/by/4.0/), which permits unrestricted use, distribution, and reproduction in any medium, provided you give appropriate credit to the original author(s) and the source, provide a link to the Creative Commons license, and indicate if changes were made.

\section{References}

1. Agrawal M, Chakrabarty K (2015) Test-Cost Modeling and Optimal Test-Flow Selection of 3-D-Stacked ICs. In: IEEE Transactions on Computer-Aided Design of Integrated Circuits and Systems, pp 1523-1536

2. Chou RM, Saluja KK, Agrawal VD (1997) Scheduling tests for VLSI systems under power constraints. IEEE Trans VLSI Syst 5(2):175-185

3. Hamdioui S, Taouil M (2011) Yield Improvement and Test Cost Optimization for 3D Stacked ICs. In: Asian Test Symposium (ATS), pp 480-485

4. Higgins M, MacNamee C, Mullane B (2010) Design and implementation challenges for adoption of the IEEE 1500 standard. IET Comput Digit Techn 4(1):38-49

5. Iyengar V, Chakrabarty K, Marinissen EJ (2002) Test Wrapper and Test Access Mechanism Co-Optimization for System-onChip. In: Journal of Electronic Testing: Theory and Applications, vol 18, pp 213-230

6. Iyengar V, Chakrabarty K, Marinissen EJ (2003) Test Access Mechanism Optimization, Test Scheduling, and Tester Data Volume Reduction for System-on-Chip. IEEE Trans Comput 52(12):1619-1632

7. Larsson E, Arvidsson K, Fujiwara H, Peng Z (2004) Efficient test solutions for core-based designs. IEEE Trans Comput-Aided Des Integr Circ Syst 23(5):758-775

8. Marinissen EJ, Zorian Y (2009) Testing 3D Chips Containing Through-Silicon Vias. In: IEEE International Test Conference (ITC), pp 1-11

9. Marinissen EJ, Verbree J, Konijnenburg M (2010) A Structured and Scalable Test Access Architecture for TSV-Based 3D Stacked ICs. In: IEEE VLSI Test Symposium (VTS), pp. 1-6

10. Marinissen EJ, McLaurin T, Jiao H (2016) Ieee std p1838: Dft standard-under-development for 2.5d-, 3d-, and 5.5d-sics. in: 2016 21th IEEE european test symposium (ETS), pp 1-10

11. Mullane B, Higgins M, MacNamee C (2008) IEEE 1500 Core wrapper optimization techniques and implementation. In: IEEE International test conference (ITC), no. 29.2, pp 1-10

12. SenGupta B, Larsson E (2014) Test Planning and Test Access Mechanism Design for Stacked Chips using ILP. In: IEEE VLSI Test Symposium (VTS), pp 1-6

13. SenGupta B, Ingelsson U, Larsson E (2012) Scheduling Tests for 3D Stacked Chips under Power Constraints. J Electron Test: Theory Appl (JETTA) 28(1):121-135

14. Taouil M, Hamdioui S (2012) Yield Improvement for 3D Waferto-Wafer Stacked Memories. In: Journal of Electronic Testing: Theory and Applications (JETTA), vol 28, pp 523-534

15. Yi H, Song J, Park S (2008) Low-Cost Scan Test for IEEE-1500Based SoC. IEEE Trans Instrum Measur 57(5):1071-1078

Publisher's Note Springer Nature remains neutral with regard to jurisdictional claims in published maps and institutional affiliations.

Breeta SenGupta received her M.Sc. degree from Indian Institute of Technology, Kharagpur, India, in 2009. She pursues her Ph.D. at the Department of Electrical and Information Technology, Lund University, Sweden. Her area of research includes 3D integration and testing. Breeta is currently engaged in development and verification of RF ASIC at Ericsson, Sweden. 
Dimitar Nikolov received the diploma engineering degree from "SS. Cyril and Methodius" University, Skopje, Macedonia, in 2008, the licentiate degree from Linköping University, Linköping, Sweden, in 2012, and the $\mathrm{PhD}$ degree from Lund University, Lund, Sweden, in 2015. From 2015, he has been a postdoc fellow with Lund University. His current research interests include fault tolerance, design and test of digital systems, computer architectures, reconfigurable computing, and digital signal processing.

Assmitra Dash received his M.Sc. degree from the department of Computer and Information Science from Linköping University in 2013, and is currently working as a software engineer in SAP SE.
Erik Larsson received his M.Sc., Tech. Lic and Ph.D from Linköping University (LiU) in 1994, 1998, 2000, respectively. After a Post Doc (2001-2002) at Nara Institute of Science and Technology (NAIST), Japan, he was with LiU as an Assistant Professor (20022005) and Associate Professor (2006-2012). Since 2012 he is with Lund University, as Associate Professor (2012-2018) and since 2018 as Professor. His current research interests include test planning for manufacturing test, test during operation (in-situ), scan-chain diagnosis, silicon debug and validation, IJTAG/SJTAG, stacked 3D chip test, fault-tolerance for MPSoCs (Multi-Processor System-onChip), and property checking in distributed systems (MPSOcS with Network-on-Chip (NoC)). He has more than 160 publications in these areas. He received the Institution of Engineering and Technology (IET) Premium Award, 2009, and best paper awards at IEEE Asian Test Symposium (ATS), 2002 and at IEEE European Test Symposium (ETS), 2016. 